This is an electronic reprint of the original article. This reprint may differ from the original in pagination and typographic detail.

Author(s): Chugh, Tinkle; Sindhya, Karthik; Miettinen, Kaisa; Jin, Yaochu; Kratky, Tomas; Makkonen, Pekka

Title: Surrogate-assisted evolutionary multiobjective shape optimization of an air intake ventilation system

Year: $\quad 2017$

Version:

Please cite the original version:

Chugh, T., Sindhya, K., Miettinen, K., Jin, Y., Kratky, T., \& Makkonen, P. (2017).

Surrogate-assisted evolutionary multiobjective shape optimization of an air intake ventilation system. In 2017 IEEE Congress on Evolutionary Computation (CEC) (pp. 1541-1548). IEEE. https://doi.org/10.1109/CEC.2017.7969486

All material supplied via JYX is protected by copyright and other intellectual property rights, and duplication or sale of all or part of any of the repository collections is not permitted, except that material may be duplicated by you for your research use or educational purposes in electronic or print form. You must obtain permission for any other use. Electronic or print copies may not be offered, whether for sale or otherwise to anyone who is not an authorised user. 


\section{Surrogate-assisted evolutionary multiobjective shape optimization of an air intake ventilation system}

\author{
Tinkle Chugh, Karthik Sindhya, Kaisa Miettinen, Yaochu Jin \\ University of Jyvaskyla \\ Faculty of Information Technology \\ FI-40014 University of Jyvaskyla, Finland \\ Email: first-name.last-name@jyu.fi
}

\author{
Tomas Kratky \\ Centre of Hydraulic Research \\ Lutin, Czech Republic \\ Email: t.kratky@sigma.cz
}

\begin{abstract}
We tackle three different challenges in solving a real-world industrial problem: formulating the optimization problem, connecting different simulation tools and dealing with computationally expensive objective functions. The problem to be optimized is an air intake ventilation system of a tractor and consists of three computationally expensive objective functions. We describe the modeling of the system and its numerical evaluation with a commercial software. To obtain solutions in few function evaluations, a recently proposed surrogate-assisted evolutionary algorithm K-RVEA is applied. The diameters of four different outlets of the ventilation system are considered as decision variables. From the set of nondominated solutions generated by K-RVEA, a decision maker having substance knowledge selected the final one based on his preferences. The final selected solution has better objective function values compared to the baseline solution of the initial design. A comparison of solutions with K-RVEA and RVEA (which does not use surrogates) is also performed to show the potential of using surrogates.
\end{abstract}

\section{INTRODUCTION}

Optimization problems involving multiple conflicting objectives are common in engineering design. Typically they have multiple optimal solutions referred to as Pareto optimal solutions with different trade-offs among objectives. A decision maker (DM) who is an expert in the application domain can determine one among them as the final solution based on her/his preference information.

In this article, we focus on addressing three major challenges which often occurr in solving real-world problems:

1) the formulation of the multiobjective optimization problem,

2) modeling of the problem and connecting different pieces of commercial simulation software for numerical evaluation and

3) dealing with computationally expensive objective functions.

The formulation of a real-world optimization problem is usually an iterative task between an expert of the problem domain and an analyst who is familiar with optimization algorithms. In the literature, most of the algorithms developed are tested on benchmark problems, where the formulation of the problem is already known. In case of real-world problems, a meaningful formulation of the optimization problem is not necessarily straightforward and may need several iterations and efforts to be able to verify the appropriateness of the formulation.

We focus on multiobjective shape optimization of a component in the air intake ventilation system of a tractor. The particular component, shown in Figure 1, is used to heat the cabin and defrost the windscreen. Here, the main goal of the $\mathrm{DM}$ is to find a design of a cooling pipe ventilation system, where the outflow of air among all outlets is the same and the pressure loss is as low as possible. For considering these conflicting goals, we formulate a multiobjective optimization problem (MOP) involving three objectives. We describe different phases of formulating the objectives and the ones that eventually were selected.

After formulating the optimization problem, the next challenge is to combine different pieces of software to obtain objective function values. In contrast to benchmark problems, where analytical forms of the objective functions are available, real-world problems can have a black-box nature and need different simulation tools or software for function evaluation. For instance, in this study, we do meshing of the geometry in one software and then export it to another one for numerical evaluation. The output generated with the numerical evaluation software is connected with the optimization algorithm. Therefore, the challenge of combining different pieces of software is relevant in real-world problems and usually not addressed in the literature in using evolutionary algorithms.

The next challenge is finding a solution with a limited computation budget. The computation time to find a solution in real-world problems like problems involving computational fluid dynamics (CFD) simulations can be substantial [1]. In such cases, one can afford only few function evaluations.

The problem considered in this article is computationally expensive and involves CFD simulations. For this problem, the computation time for one function evaluation on a computer with Intel Xeon CPU E5-1607 v3 and 32 GB RAM is three to five minutes. To address the challenge of a limited computation budget, one can employ surrogates (or metamodels or response surface approximations) with evolutionary algorithms. Several algorithms have been proposed in the literature, e.g. ParEGO [2], SMS-EGO [3] and MOEA/D-EGO [4] to obtain a set of solutions in few function evaluations. For more details about these algorithms and their main characteristics, see [5], [6]. 
Recently, Chugh et al. [7] have proposed a surrogateassisted algorithm called K-RVEA for computationally expensive optimization problems involving three or more objective functions and demonstrated its efficiency. Kriging models have been popular in solving computationally expensive MOPs [8], [9] and are also used in K-RVEA to alleviate the computational cost of evaluating objective functions. The main focus in $\mathrm{K}$ RVEA is to efficiently manage the surrogates and reduce the training time of the surrogates. The algorithm uses a set of reference vectors for managing the surrogates to obtain a diverse set approximating Pareto optimal solutions. The potential of the algorithm was shown in [7] on several benchmark problems by comparing with the state-of-the-art surrogateassisted evolutionary algorithms.

We divide the consideration of the shape optimization problem into three phases. In the first phase, we focus on formulating the multiobjective optimization problem. In the next phase, we apply K-RVEA to find a representative set of Pareto optimal solutions. In the final phase, the DM who is the design expert in the automobile industry selects one solution of the set based on his preferences after a careful investigation.

The rest of this article is organized as follows. In Section II, we introduce some relevant characteristics of the air intake ventilation system in a tractor. Next, in Section III, we describe details and challenges occurred in formulating the optimization problem. In Section IV, we describe the modeling and numerical evaluation of objective functions with two pieces of commercial software. We briefly outline the K-RVEA algorithm used for solving the problem in Section $\mathrm{V}$ and present the results obtained in Section VI. Finally, in Section VII we draw conclusions.

\section{AIR INTAKE VENTILATION SYSTEM}

In this section, we introduce the use of an air intake ventilation system in a tractor. The air intake is a part of a ventilation system in a tractor cabin and plays a crucial role in maintaining a uniform temperature. As can be seen in Figure 1, the component of the system considered in this study consists of four outlets leading to different branches of the ventilation system. Each outlet has its own role in maintaining the temperature. In an ideal scenario, flow rates from all these outlets should be the same to provide the best uniformity in the temperature. However, maintaining an equal flow rate from each outlet is not possible in practice as each outlet has a different shape and consequently, has a different hydraulic resistance.

For any component, the pressure loss $\Delta P$ in a passage is defined as

$$
\Delta P=\Delta P_{\text {friction }}+\Delta P_{\text {local }}
$$

where $\Delta P_{\text {friction }}$ is the pressure loss caused by the friction resistance and $\Delta P_{\text {local }}$ is the pressure loss caused by local resistance. The friction resistance $\Delta P_{\text {friction }}$ occurs because of a momentum transfer to the solid walls [10]. Its numerical value can be estimated by Darcy's empirical formula:

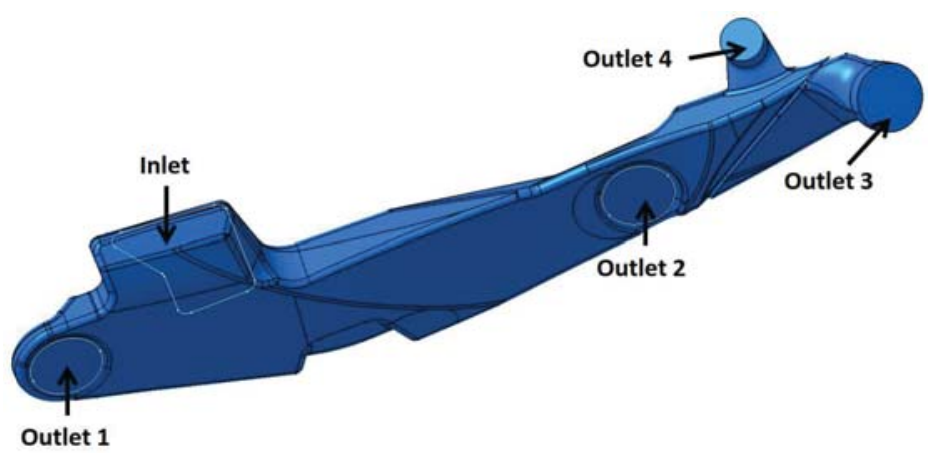

Fig. 1. CATIA 3D model of the component considered

$$
\Delta P_{\text {friction }}=\bar{f} \frac{l}{D_{H}} \frac{\rho \bar{u}^{2}}{2},
$$

where $\bar{f}$ is the Moody friction factor, $l$ and $D_{H}$ are the length and the hydraulic diameter of the passage, respectively, $\rho$ is the fluid density and $\bar{u}$ is the mean velocity of the flow.

The local resistance $\Delta P_{\text {local }}$ is due to the dissipation of mechanical energy (by whirls etc.), and can be described by the Weisbach formula:

$$
\Delta P_{\text {local }}=\zeta \frac{\rho \bar{u}^{2}}{2}
$$

where $\zeta$ is the coefficient of local resistance.

In fact, the coefficients $\bar{f}$ and $\zeta$ are functions of both the passage shape and the Reynold's number. The dimensionless Reynold's number Re represents the ratio between momentumrelated force and viscous shear force, and is defined in [11] as:

$$
R e=\frac{u L \rho}{\eta},
$$

where $u$ is the fluid velocity, $\rho$ the fluid density, $\eta$ the fluid viscosity and $L$ the so-called characteristic length. The values of the hydraulic resistance coefficients $\bar{f}$ and $\zeta$ can be obtained by multiple means: experimentally or numerically through CFD. For many "standard" shapes, they can also be found in the literature, e.g. in [10].

Generally, the pressure loss increases with an increasing velocity. This is because of the quadratic member $\rho \bar{u}^{2}$ in (2) and (3). Now that some basic concepts of the ventilation system have been introduced, we can formulate the multiobjective optimization problem to be considered.

\section{MOP FORMULATION}

In this section, we first discuss the challenges occurred in formulating the multiobjective optimization problem and then present the objective functions formulated. 


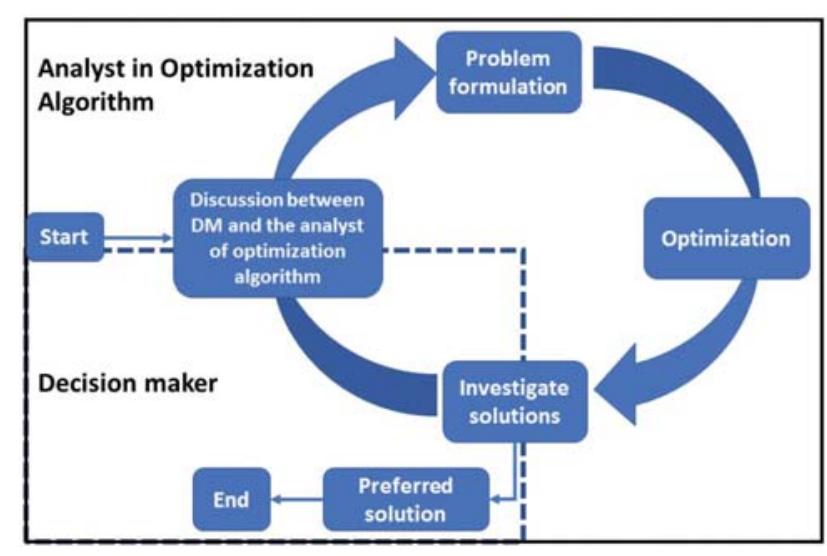

Fig. 2. An illustration of the optimization problem formulation in real-world problems

\section{A. Challenges in formulating the problem}

As mentioned in the introduction, formulating a meaningful representation of the optimization problem which reflects the needs and is understandable for the DM is not necessarily straightforward. It is very important to mention this particular challenge as most of the evolutionary algorithms in the literature are tested on benchmark problems, where no effort for the formulation of the optimization problem is needed. A typical illustration of the formulation and solving a real-wold optimization problem is presented in Figure 2. To summarize, in this study, first, a problem was formulated after an initial discussion with the expert who was the DM in the application domain and the optimization analyst. After solving the problem, the solutions were shown to the DM and the problem was revised and reformulated e.g. by adding, removing and modifying the objective functions. After these several iterations, a final formulation was selected.

We finalized the formulation after three iterations with the DM. First, we formulated a five objective optimization problem. Solutions obtained after solving this problem were not providing any extra value in terms of objective functions to the DM. Therefore, we modified the problem to have two objectives. Solutions of this biobjective optimization problem were meaningful to the DM and were also providing better values in terms of objective functions e.g. a good balance in the flow rates from different outlets and low pressure losses. However, the flow rate from one of the outlets was very low because of its small diameter. Therefore, we reformulated the problem to have three objectives by adding an extra objective considering the flow rate from the outlet with the smallest diameter. The DM was satisfied with the solutions and finally selected one solution based on his preferences. Next, we define the objective functions and decision variables of the final formulation of the multiobjective optimization problem.

\section{B. Objective functions and decision variables}

As mentioned in Section II, the pressure loss depends on hydraulic resistance and the mean velocity $\bar{u}$. All the outlets

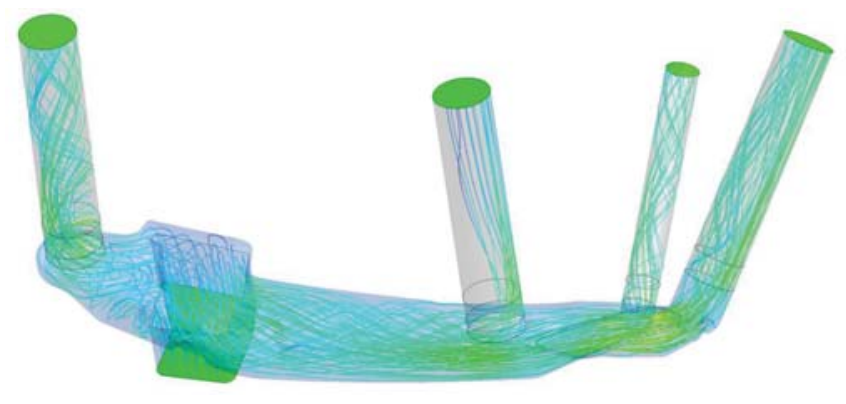

Fig. 3. CFD results of the initial design

open into a surrounding with the same pressure. Therefore, the pressure loss, i.e. the difference of pressures at inlet and outlet, must be the same for all the outlets. If the hydraulic resistances of different outlets are not the same, the velocities and thus the flow rates must be different according to equations (2) and (3). Also, flow rates from outlets 2 and 4 are significantly lower when compared to other outlets. This is mainly caused by the characteristic of the flow in case of outlet 2 , and by the small diameter (small diameter leads to a high velocity) of outlet 4 . A CFD simulation of the initial design given by the DM is shown in Figure 3. In the following, we denote the solution corresponding to the initial design as the baseline solution.

Maximizing the balance in the flow rates and minimizing pressure losses are two major goals in the optimization. As the flow rates are different from different outlets because of the hydraulic resistances (the higher the resistance, the smaller the flow rate), a balance in the flow rate can be accomplished in two ways:

1) decreasing the high hydraulic resistances or

2) increasing the low hydraulic resistances.

Since the pressure loss is mostly determined by the narrowest parts of the passage, the diameters of the outlets play a key role. A smaller diameter leads to a high hydraulic resistance and vice-versa. Decreasing the hydraulic resistances would be good for both balances in the flow rates and reducing the pressure losses. Therefore, the diameters of the outlets can be used as the decision variables. To ease the solution process, we use the scaling factors of the initial design as the decision variables i.e.

$$
x_{i}=\frac{D_{i}}{D_{i}^{(\text {initial })}} \text { for } i=1, \ldots, 4,
$$

where $D_{i}$ is the diameter of the $i^{\text {th }}$ outlet and $D_{i}^{\text {initial }}$ is the diameter of the $i^{\text {th }}$ outlet in the initial design. The diameters of the outlets were also prolonged as shown in Figure 3 for numerical evaluation using CFD simulations. This was easily accomplished using the custom $T c l$ scripts of ANSYS ICEM. Such a simplification is only accurate if changes in diameters are not greater than the original ones. However, for the solution process, we used the following bounds of the decision variables:

$$
\begin{aligned}
& x_{i}^{l b}=0.5 \text { for } i=1, \ldots, 4, \\
& x_{i}^{u b}=1.5 \text { for } i=1, \ldots, 4,
\end{aligned}
$$


where $x_{i}^{l b}$ and $x_{i}^{u b}$ are the lower and upper bounds of the decision variables.

In addition to maximizing the balance in flow rates and minimizing the pressure losses, a third objective considering the flow rate from the outlet 4 was considered because of its smallest diameter. Finally, we finalized the following three objectives:

$f_{1}$ : Minimize variance between flow rates at outlets 1 to 3

: Minimize $\operatorname{var}\left(Q_{1,3}\right)$

$f_{2}$ : Minimize pressure loss of the air intake

: Minimize $P_{\text {inlet }}-P_{\text {outlet }}$

$f_{3}$ : Minimize the difference between the flow rate at outlet 4 and the average of the flow rates at outlets 1 to 3

: Minimize $\operatorname{avg}\left(Q_{1,3}\right)-Q_{4}$,

where $Q_{k}$ represents the flow rate from the $k^{\text {th }}$ outlet, $\operatorname{avg}\left(Q_{1,3}\right)$ the average flow rate value from outlets 1-3 and $P_{\text {inlet }}$ and $P_{\text {outlet }}$ are the pressure values at the inlet and the outlet, respectively. As already mentioned, decision variables are the scaling factors of the diameters of the initial design given in (5).

For numerical evaluation of the designs, the ANSYS CFX [12] solver was used for CFD simulations. For some standard shapes such as elbows or pipes, it would be easier to use empirical values for hydraulic resistance coefficients. But in this particular case, CFD takes into account both the exact shape of the design and a mutual interaction of the outlets. This allows for a higher precision in the numerical evaluation. Next, we provide a detailed description of modelling and numerical evaluation.

\section{Modelling AND NUMERICAL EVALUATION}

For the CFD analysis, a three dimensional model of the component was built in the modelling software CATIA [13] as shown in Figure 4. The model was then exported to ANSYS ICEM [14] for meshing and a tetra mesh with prism layers was created. For a better numerical stability, the inlet and outlet parts were extended as shown in the figure (the gray zones). However, these extensions could cause differences in the relative flow rates, and therefore they were considered as free-slip walls. The boundary conditions were set as Mass Flow at the inlet and Relative Pressure at the outlets, with a $\mathrm{k}-\epsilon$ turbulence model.

The parametric model was realized using ANSYS ICEM, ANSYS CFX and custom scripts. For the sake of simplicity, the original CATIA model and the mesh were fixed in the parametric model. Changing the diameters was instead approximated by changing the diameters of the extended outlet parts. This approximation was easily realized through ICEM Tcl scripts and gave accurate results for $x_{i} \leq 1$ (because the pressure loss mostly depends on the narrowest part). For $x_{i}>1$, the fixed part of the model plays an important role and increasing $x_{i}$ in such a case does not decrease the pressure loss significantly.

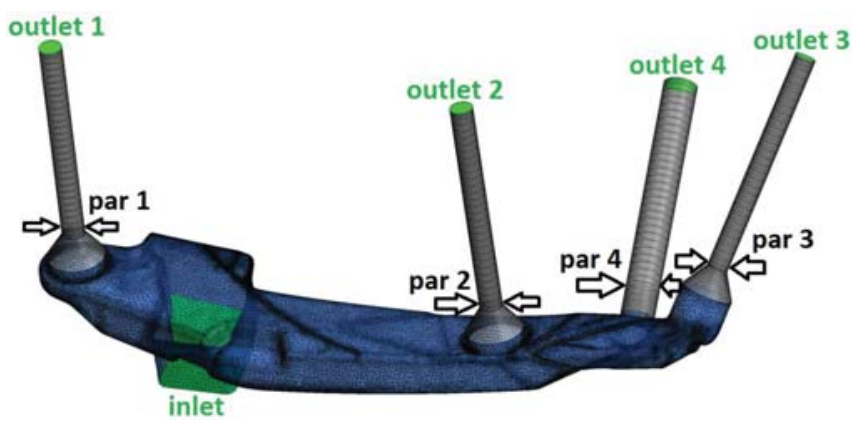

Fig. 4. Parametric CFD model

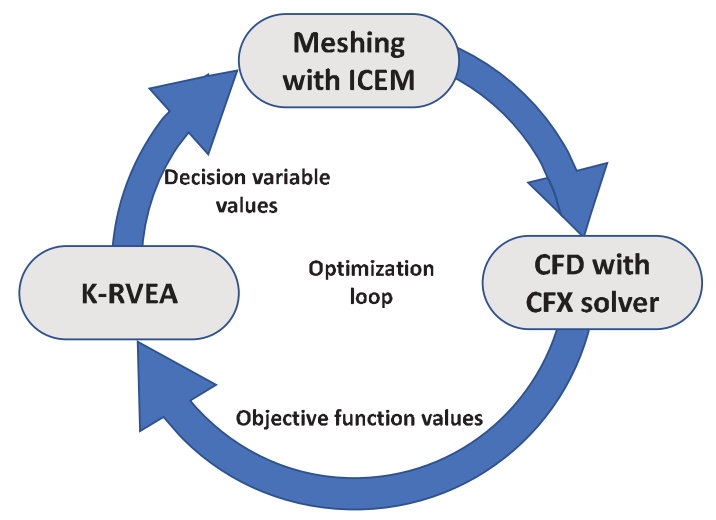

Fig. 5. Illustration of the optimization loop

As mentioned, outlet 4 has the smallest diameter as can be seen in Figure 4 and achieving a high flow rate from this outlet is difficult. Therefore, a special attention was paid towards this outlet while formalizing the objective functions. The mesh generated using ICEM was then exported to CFX for CFD simulations. When using the CFX tools, the values of pressure loss and flow rates were recorded. In order to automate the process during optimization, different scripts were written in Python and Matlab to connect different tools and optimization algorithm.

An illustration of the optimization loop used in this study is shown in Figure 5. First, the meshing is done for a given shape of the component using ANSYS ICEM. After meshing, CFD simulations are performed with the solver ANSYS CFX. Based on this numerical evaluation, K-RVEA produces new values for the decision variables and a new shape is created which then goes for meshing. The loop continues for a prefixed number of evaluations and nondominated solutions of all the evaluated ones are used as the final set of solutions. Next, we provide a brief outline of K-RVEA which was used to solve the problem formulated.

\section{Surrogate-Assisted evolutionary algorithm}

The K-RVEA algorithm [7] has been developed for computationally expensive many-objective optimization problems. 
However, the efficiency of the algorithm was also demonstrated on three objective optimization problems in [7]. The algorithm uses elements from its underlying evolutionary algorithm RVEA [15] and Kriging models as surrogates. It consists of three phases as presented in Algorithm 1. Nondominated solutions from all the evaluated ones stored in an archive $A 2$ are used as final solutions.

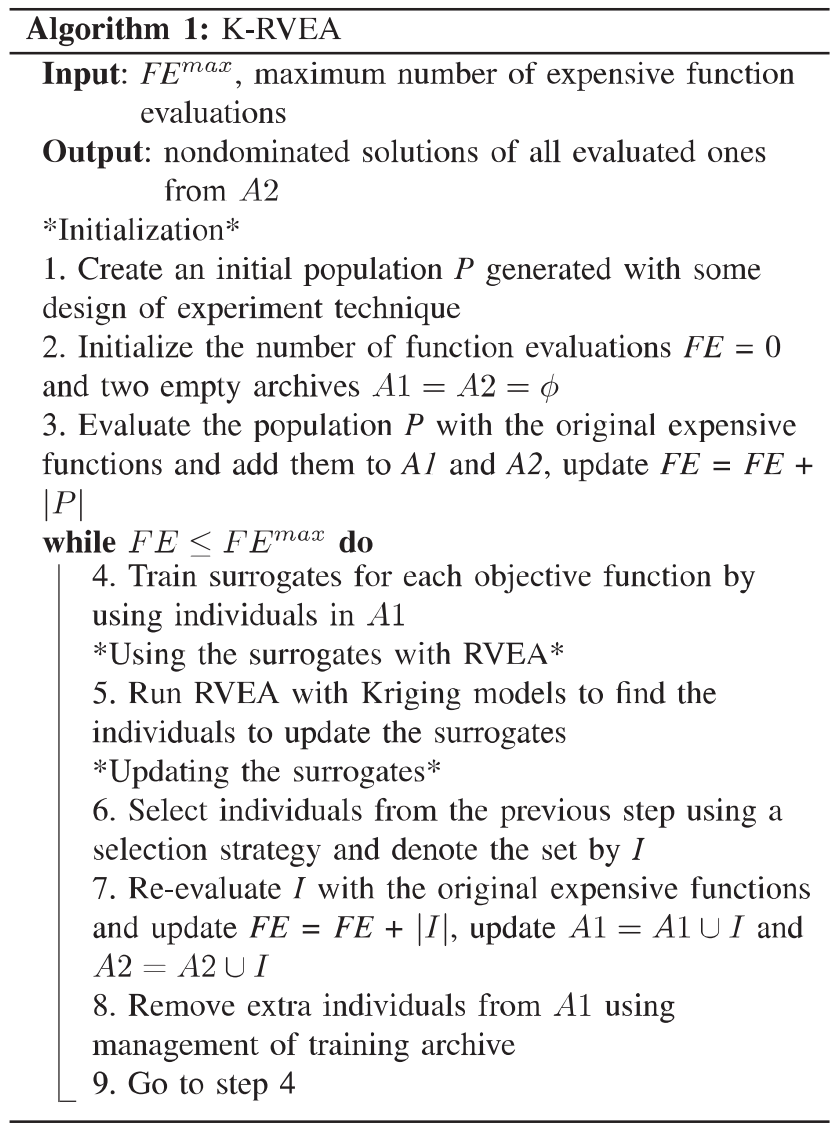

In the initialization phase, an initial population is generated e.g. using the Latin hypercube sampling [16]. This population is then evaluated with the original expensive functions and added to two archives $A 1$ and $A 2$. Individuals in $A 1$ are used to build surrogates for each objective function in step 4 . The archive $A 2$ is used to store all the evaluated solutions.

When using the surrogates with RVEA [15], we use mean values of Kriging models as objective function values. In addition, uncertainty information of the approximated solutions is obtained which is further used in updating the surrogates. Solutions generated are then used to select individuals for reevaluation with the original expensive objective functions for updating the surrogates.

Updating the surrogates is very important in model management [6], [17]. Individuals for updating the surrogates should be selected in such a way that they enhance both convergence and diversity. In K-RVEA, these two criteria are taken care of with the help of reference vectors and the

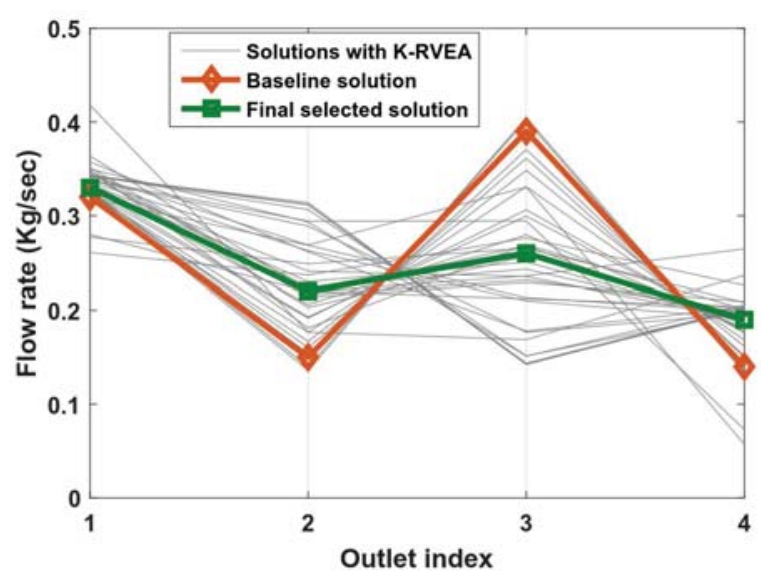

Fig. 7. Flow rates corresponding to nondominated solutions

uncertainty information from the Kriging models. Individuals with a maximum uncertainty are selected whenever diversity is needed. If a satisfactory degree of diversity has already been achieved, individuals with a minimum angle penalized distance are selected, which is one of the selection criteria in RVEA that contributes to convergence. Once these individuals have been selected, they are re-evaluated with the original expensive functions and added to archives $\mathrm{A} 1$ and $A 2$. As the size of the data set can influence the training time, to decrease the computation time further, some of the individuals are removed from $A 1$ in step 8 . To be able to do that, reference vectors are used to identify the extra individuals which were not needed for training the surrogates. The individuals in $A 1$ are then used to re-train the surrogates in step 4 and the algorithm is run until a maximum number of expensive function evaluations has been reached. For full details about the selection strategy and the management of the training archive, see [7]. Next, we present the results obtained with K-RVEA on the given problem.

\section{RESULTS AND DISCUSSION}

In this section, we report the results and an analysis of the solutions obtained with K-RVEA. Parameters used are given in Table I, where $n$ represents the number of decision variables. Also, note that we ran the optimization solution process only for one run due to a limited computation budget.

TABLE I

PARAMETER VALUES USED

\begin{tabular}{|l|c|}
\hline Parameter & Value \\
\hline Number of function evaluations & 200 \\
\hline Size of the training data set & $11 n-1$ \\
\hline Number of reference vectors & 105 \\
\hline
\end{tabular}



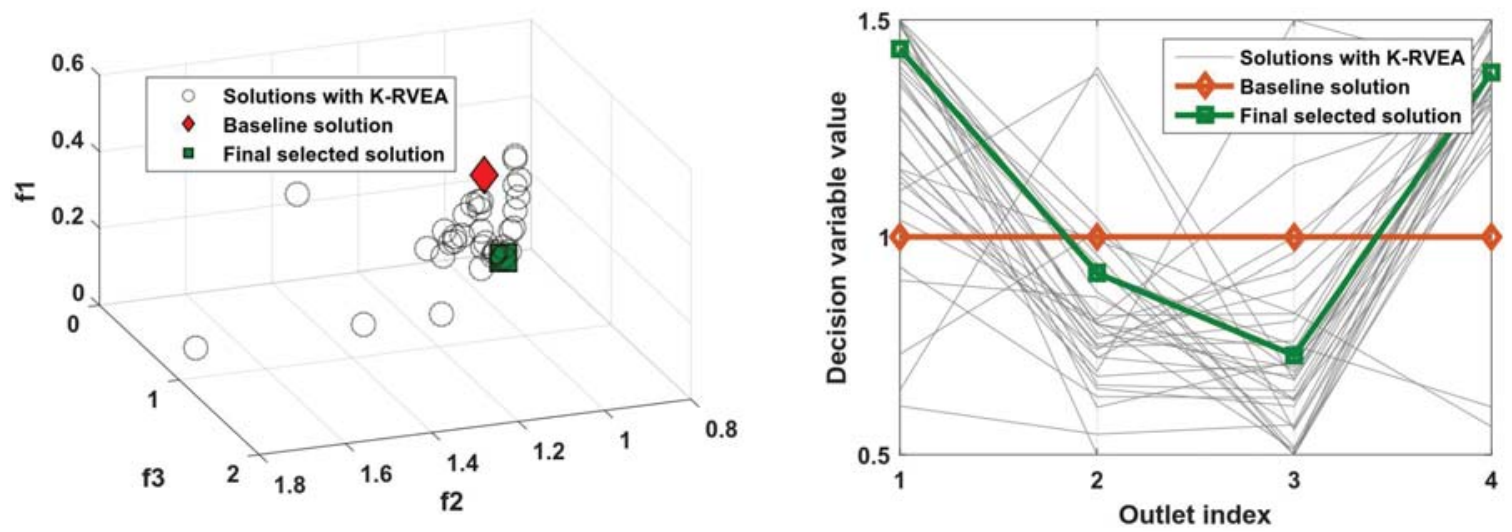

Fig. 6. Nondominated solutions in the objective (normalized) and decision spaces

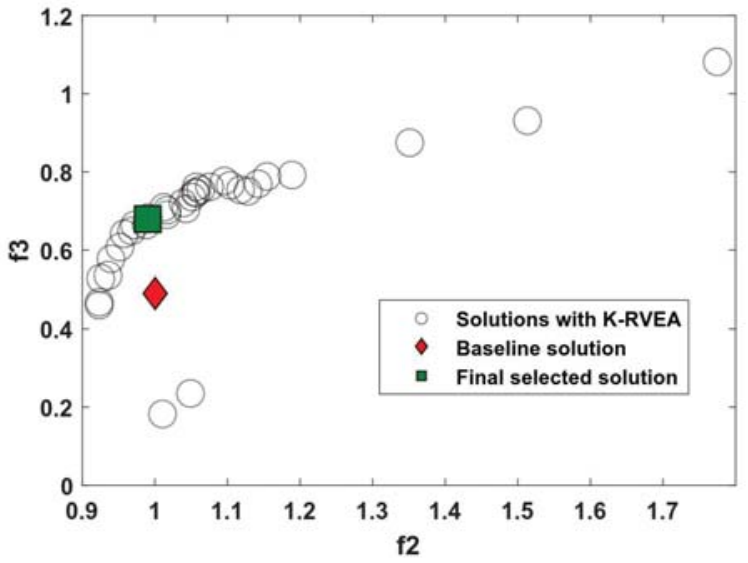

Fig. 8. Projection of nondominated solutions for the second and the third objectives

To protect confidential data, objective function values obtained are normalized in the following way:

$f_{1}: \frac{\sum_{k=1}^{3}\left|Q_{k}-\operatorname{avg}\left(Q_{1,3}\right)\right|}{3 * \operatorname{avg}\left(Q_{1,3}\right)}$

$f_{2}: \frac{P_{\text {inlet }}-P_{\text {outlet }}}{P_{\text {original }}}$

$f_{3}: \frac{Q_{4}}{\operatorname{avg}\left(Q_{1,3}\right)}$,

where $P_{\text {original }}$ is the absolute pressure value of the initial geometry.

Nondominated solutions obtained are shown in Figure 6 in the objective and decision spaces. For a given pressure loss, a better balance in the flow rates from outlets 1-3 was obtained. However, pressure losses were high for a high flow rate from the outlet 4 . This is due to the fact that outlet 4 has a very small diameter when compared to the other outlets. Solutions are also compared with the baseline solution of the initial geometry as shown in Figure 6. Many solutions obtained with K-RVEA dominate the baseline solution.

As can be seen in the decision space, the algorithm tries to find solutions with a high diameter especially for outlets 1 and 4. This is due to the reason that in the initial geometry, outlet 4 had the smallest diameter and increasing it was the only option of increasing $Q_{4}$. The outlet 1 might seem a bit counterintuitive at first, because in the baseline solution $Q_{1}>Q_{2}$. We assume this is caused by pursuing the minimization of pressure loss and the complexity of the flow, where each outlet can slightly influence the other ones.

We also present the corresponding flow rates from all four outlets in Figure 7. These flow rates are normalized as follows:

$$
Q_{k}=\frac{Q_{k}}{Q_{\text {input }}}, \text { for } k=1, \ldots, 4,
$$

where $Q_{\text {input }}$ is the flow rate at the inlet of the component. It can be seen that for the baseline solution, flow rate values from different outlets were very different from each other and maintaining a uniform temperature was not easily achievable. K-RVEA produced a diverse set of flow rate values as shown in the figure except for outlets 1 and 4 . The reason for this is the same as mentioned above i.e. a small diameter for outlet 4 and a low pressure loss from outlet 1 .

A projection of the solutions obtained for the second and third objectives is shown in Figure 8. It can be clearly observed the $f_{2}$ and $f_{3}$ are conflicting objectives, i.e. a high flow rate from outlet 4 causes high pressure losses and consequently, the flow rates from other outlets are decreased. In other words, the diameters for outlets 1-3 decrease less for a high flow rate from outlet 4 . For $f_{3} \geq 0.46$, all four outlets were active or in other words, all the outlets were fully open. For $f_{3}<0.46$, outlets 1-3 were inactive and pressure losses were increased. This is the reason why two solutions were not contributing to the trade-off between the second and the third objective.

Finally, 40 solutions were shown to the DM and he selected his most preferred solution. The baseline solution and the solution selected by the DM are shown in Figures 6, 7 and 8. As can be seen, the results confirm the original assumptions 


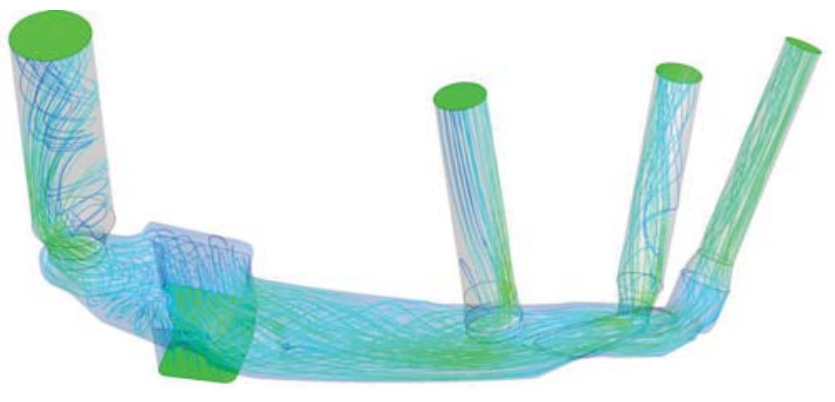

Fig. 9. The design of the final solution selected by the DM

of the necessary of trade-offs between flow rates and pressure loss. Considering the flow rate from outlet 4 as an additional objective did also prove to be a useful decision.

The CFD simulation of the final solution selected by the DM is shown In Figure 9. The solution has a very small variance between flow rates from outlets 1-3 for an equivalent pressure loss compared to the baseline solution. Also, the flow rate from outlet 4 is comparable to flow rates from other outlets. As the balance in flow rates has been increased in the final selected solution, more air flow can be delivered into the cabin using the same fan. This will improve the cooling and heating properties of the cabin which usually improves customer satisfaction.

The DM was happy with the solution obtained. However, out of academic interest, we also compared the solutions of the single run of K-RVEA and RVEA to show the efficiency of using surrogates. We ran RVEA for the same number of function evaluations. The nondominated solutions obtained with both algorithms in the objective space are shown in Figure 10. As can be seen, K-RVEA produced a better distribution of solutions than RVEA. The hypervolume with the number of function evaluations for both algorithms is presented in Figure 11. We used the worst objective function values from nondominated solutions of both algorithms as a reference point $\left(f_{i}^{*}\right)$ in calculating the hypervolume. The values obtained were normalized by dividing with $\prod_{i=1}^{k} f_{i}^{*}, k$ being the number of objectives. For a given number of function evaluations, KRVEA performed better than RVEA in terms of the hypervolume. We also used coverage [18] and inverted generational distance (IGD) [19] as other two performance metrics to compare the results. The values of all three performance metrics after 200 function evaluations are given in Table II. The coverage metric is usually used to compare solutions based on their dominance. For instance, in Table II, 0.5152 means that $51.52 \%$ of the solutions with RVEA are dominated by the ones with K-RVEA. To calculate IGD, we combined nondominated solutions from both algorithms and used this union as the reference set in calculating the values. In all these performance metrics, K-RVEA performed better than RVEA which shows that using surrogates efficiently with evolutionary algorithms can be helpful in getting better quality solutions with a limited computation budget. Comparing K-RVEA with

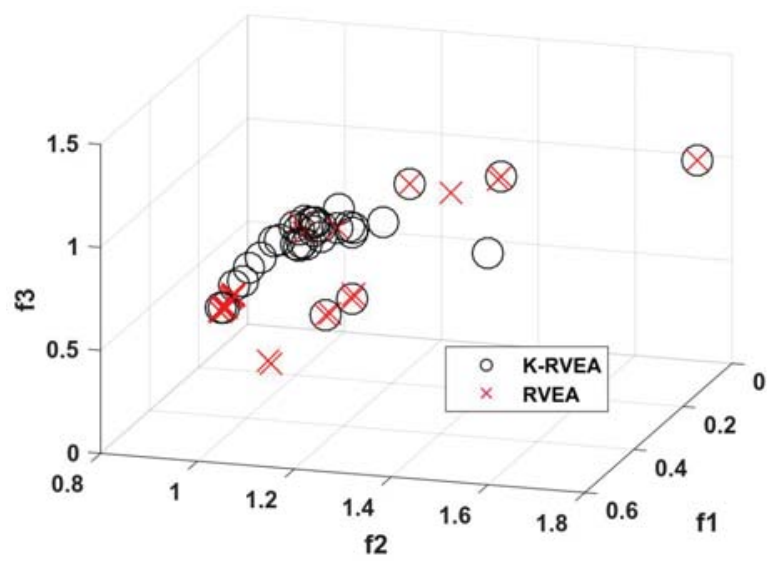

Fig. 10. Nondominated solutions with algorithms K-RVEA and RVEA

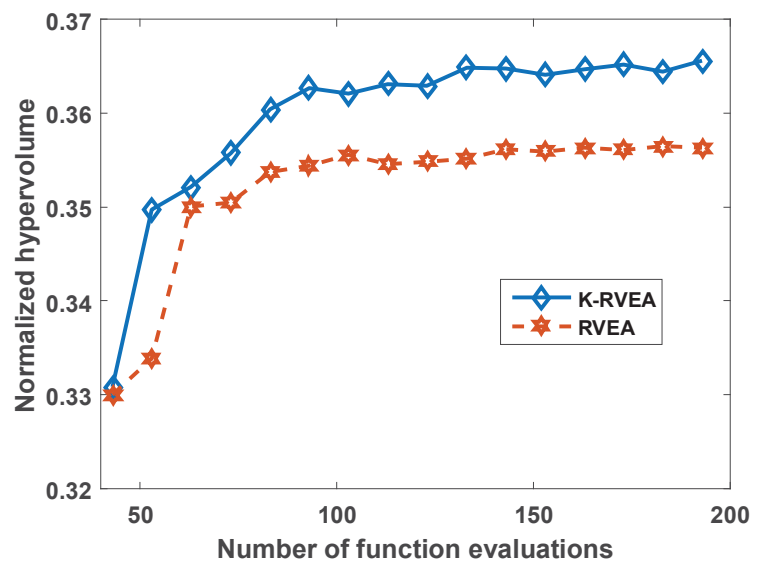

Fig. 11. Normalized hypervolume with the number of function evaluations in a single run

other surrogate-assisted evolutionary algorithms will be our future work.

TABLE II

HYPERVOLUME, COVERAGE AND IGD OF SOLUTIONS WITH K-RVEA AND RVEA

\begin{tabular}{|c|c|c|c|}
\hline Algorithm & Hypervolume & Coverage & IGD \\
\hline K-RVEA & $\mathbf{0 . 3 6 5 6}$ & $\mathbf{0 . 5 1 5 2}$ & $\mathbf{0 . 0 8 8 1}$ \\
\hline RVEA & 0.3563 & 0.2059 & 0.3320 \\
\hline
\end{tabular}

\section{Conclusions}

A multiobjective shape optimization problem of an air intake ventilation system of a tractor with four outlets was formulated, implemented and solved. Thus, three main challenges in solving real-world problems, formulating the optimization problem, combining optimization and different simulation tools and dealing with computationally expensive objective 
functions were emphasized. A description about the modelling of the given problem and solving with a CFD solver was also detailed. To alleviate the computational cost, a recently proposed surrogate-assisted evolutionary algorithm K-RVEA was applied. It was selected as it had shown a very good performance when compared to other surrogate assisted algorithms. A diverse set of solutions representing the balance in flow rates from outlets 1-3, low pressure losses and a high flow rate from outlet 4 were obtained. Among the solutions obtained, a final solution was selected by the DM. The selected solution is significantly better in two objectives and similar in the third objective compared to the baseline solution.

To show the efficiency of K-RVEA, solutions were also compared with its underlying evolutionary algorithm RVEA. K-RVEA performed better than RVEA in terms of hypervolume, coverage and IGD which shows the benefits of using surrogates in dealing with (computationally expensive) realworld problems. Additionally, practitioners in industry usually face the challenges of formulating the optimization problem and connecting different pieces of simulation tools. Therefore, the current approach by focusing on these two challenges and solving the formulated MOP having computationally expensive objective functions may be helpful in providing insight to formulate the optimization problem and solving it with an appropriate algorithm.

In this article, all the nondominated solutions generated were shown to the DM. However, a better way of interacting with the DM with a good visualization of solutions is needed. As the problem is computationally expensive, interacting with the DM may also save the computational resources when not all nondominated solutions are of interest. Thus, developing an interactive algorithm using surrogates will be our future work.

\section{ACKNOWLEDGMENT}

This work was funded by TEKES, the Finnish Funding Agency for Innovation under the FiDiPro project DeCoMo. We would also like to thank Valtra Inc. for providing the problem.

\section{REFERENCES}

[1] M. Emmerich and B. Naujoks, "Metamodel assisted multiobjective optimisation strategies and their application in airfoil design," in Adaptive Computing in Design and Manufacture VI, I. C. Parmee, Ed. Springer London, 2004, pp. 249-260.

[2] J. Knowles, "ParEGO: a hybrid algorithm with on-line landscape approximation for expensive multiobjective optimization problems," IEEE Transactions on Evolutionary Computation, vol. 10, pp. 50-66, 2006.

[3] W. Ponweiser, T. Wagner, D. Biermann, and M. Vincze, "Multiobjective optimization on a limited budget of evaluations using model-assisted S-metric selection," in Proceedings of the Parallel Problem Solving from Nature-PPSN X, G. Rudolph, T. Jansen, S. Lucas, C. Poloni, and N. Beume, Eds. Springer, Berlin, Heidelberg, 2008, pp. 784-794.

[4] Q. Zhang, W. Liu, E. Tsang, and B. Virginas, "Expensive multiobjective optimization by MOEA/D with Gaussian process model," IEEE Transactions on Evolutionary Computation, vol. 14, pp. 456-474, 2010

[5] T. Chugh, K. Sindhya, J. Hakanen, and K. Miettinen, "Handling computationally expensive multiobjective optimization problems with evolutionary algorithms - A survey. Reports of the Department of Mathematical Information Technology, Series B, Scientific Computing no. B 4/2015, University of Jyvaskyla," 2015.
[6] Y. Jin, "Surrogate-assisted evolutionary computation: Recent advances and future challenges," Swarm and Evolutionary Computation, vol. 1, no. 2, pp. 61-70, 2011.

[7] T. Chugh, Y. Jin, K. Miettinen, J. Hakanen, and K. Sindhya, "A surrogate-assisted reference vector guided evolutionary algorithm for computationally expensive many-objective optimization," IEEE Transactions on Evolutionary Computation, to appear, doi:10.1109/TEVC.2016.262230

[8] J. Shinkyu, Y. Minemura, and S. Obayashi, "Optimization of combustion chamber for diesel engine using Kriging model," Journal of Fluid Science and Technology, vol. 1, pp. 138-146, 2006

[9] A. Forrester and A. Keane, "Recent advances in surrogate-based optimization," Progress in Aerospace Sciences, vol. 45, pp. 50-79, 2009.

[10] I. E. Idelchik, Handbook of Hydraulic Resistance. Jaico Publishing House, 2005

[11] R. A. Granger, Fluid Mechanics. Dover Publishing House, 1985.

[12] N. Trev, CFX: Computational Fluid Dynamics, Ansys, HVAC. International Book Market Service Limited, 2012.

[13] S. Tickoo, CATIA V5R17: For Engineers and Designers. Dreamtech Press, 2008.

[14] ANSYS ICEM CFD Tutorial Manual, ANSYS, Inc., 2013.

[15] R. Cheng, Y. Jin, M. Olhofer, and B. Sendhoff, "A reference vector guided evolutionary algorithm for many-objective optimization," IEEE Transactions on Evolutionary Computation, vol. 20, pp. 773-791, 2016.

[16] M. Mckay, R. Beckman, and W. Conover, "A comparison of three methods for selecting values of input variables in the analysis of output from a computer code," Technometrics, vol. 42, pp. 55-61, 2000.

[17] Y. Jin, "A comprehensive survey of fitness approximation in evolutionary computation," Soft Computing, vol. 9, pp. 3-12, 2005.

[18] E. Zitzler and L. Thiele, "Multiobjective evolutionary algorithms: A comparative case study and the strength pareto approach," IEEE Transactions on Evolutionary Computation, vol. 3, pp. 257-271, 1999.

[19] P. Bosman and D. Thierens, "The balance between proximity and diversity in multiobjective evolutionary algorithms," IEEE Transactions on Evolutionary Computation, vol. 7, pp. 174-188, 2003. 